\title{
Atheroprotective effects of methotrexate via the inhibition of YAP/TAZ under disturbed flow
}

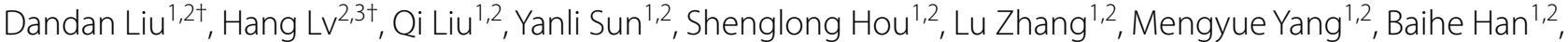 \\ Gang wang ${ }^{1,2}$, Xuedong Wang ${ }^{1,2}$, Wenjuan Du ${ }^{1,2}$, Honggang Nie ${ }^{1,2}$, Ruoxi Zhang ${ }^{1,2}$, Xingtao Huang ${ }^{1,2}$, \\ Jing bo Hou ${ }^{1,2^{*}}$ and $\mathrm{Bo} \mathrm{Yu}^{1,2}$
}

\begin{abstract}
Background: Atherosclerosis preferentially develops in regions of disturbed flow (DF). Emerging evidence indicates that yes-associated protein (YAP) and transcriptional co-activator with PDZ-binding motif (TAZ), which are both effectors of the Hippo pathway, sense different blood flow patterns and regulate atherosclerotic lesions. We previously found that methotrexate (MTX) reduces in-stent neoatherosclerosis, decreases the plaque burden, and has an effect on local fluid shear stress. Here, we investigated the atheroprotective effect of MTX under DF and the mechanisms underlying these properties.

Methods: Human umbilical vein endothelial cells (HUVECs) were subjected to biomechanical stretch using a parallel-plate flow system and treated with or without MTX at therapeutically relevant concentrations. Additionally, an extravascular device was used to induce DF in the left common carotid artery of C57BL/6 mice, followed by treatment with MTX or $0.9 \%$ saline. The artery was then assessed histopathologically after 4 weeks on a Western diet.

Results: We observed that MTX significantly inhibited DF-induced endothelial YAP/TAZ activation. Furthermore, it markedly decreased pro-inflammatory factor secretion and monocyte adhesion in HUVECs but had no effect on apoptosis. Mechanistically, AMPKa1 depletion attenuated these effects of MTX. Accordingly, MTX decreased DFinduced plaque formation, which was accompanied by YAP/TAZ downregulation in vivo.
\end{abstract}

Conclusions: Taken together, we conclude that MTX exerts protective effects via the AMP-dependent kinase (AMPK)YAP/TAZ pathway. These results provide a basis for the prevention and treatment of atherosclerosis via the inhibition Of YAP/TAZ.

Keywords: Methotrexate, Human umbilical vein endothelial cells, YAP/TAZ, Shear stress, AMP-dependent kinase

\section{Background}

Atherosclerosis is currently the leading cause of mortality worldwide [1, 2]. Despite advances in disease-modifying and biological therapy for the disease, specific strategies aimed at retarding its development are lacking, and the knowledge of whether individual drugs offer vascular

\footnotetext{
*Correspondence: jingbohou@163.com

${ }^{+}$Dandan Liu and Hang Lv contributed equally to this paper

${ }^{1}$ Division Department of Cardiology Organization, The Second Affiliated

Hospital of Harbin Medical University, Harbin, China

Full list of author information is available at the end of the article
}

protection is limited. Atherosclerotic lesions develop in the arteries at sites of disturbed flow (DF) and shear stress plays a critical role in plaque location and progression [3-5]. Recent research has demonstrated that systemic inhibition of the Hippo pathway effectors yesassociated protein (YAP) and transcriptional co-activator with PDZ-binding motif (TAZ) attenuates the development of atherosclerotic lesions induced by DF [6]. YAP/ TAZ responds to haemodynamic forces and transduces mechanical signals into chemical signals [7]. DF promotes YAP/TAZ activation and dephosphorylation, and the dephosphorylated form of YAP is translocated from 
the cytoplasm to the nucleus to up-regulate target genes including cysteine-rich angiogenic inducer 61 (CYR61) and connective tissue growth factor (CTGF), and to stimulate pro-inflammatory gene expression, thereby increasing monocyte attachment and infiltration, which contribute to atherogenesis $[6,8]$.

Several studies have demonstrated that long-term lowdose methotrexate (MTX) therapy in rheumatoid arthritis is associated with reduced cardiovascular disease and cardiovascular mortality $[9,10]$. Likewise, MTX-treated animals show reduced rates of lipid-rich intima [11]. MTX increases the intracellular accumulation of adenosine monophosphate (AMP) and 5-aminoimidazole4-carboxamide ribonucleotide (AICAR), which activates AMP-activated protein kinase (AMPK) $[12,13]$. AMPK plays a role in promoting YAP phosphorylation at Ser127, which phosphorylates YAP to induce its cytoplasmic localization and proteasomal degradation; therefore, AMPK activation results in YAP phosphorylation and inactivation [14-16]. We hypothesised that the AMPK pathway mediates YAP/TAZ functional inactivation and the atheroprotective effects of MTX.

Therefore, in this study, the contribution of MTX to atheroprotective effects and the signalling mechanism underlying such protective effects against DF were evaluated.

\section{Materials and methods}

\section{Materials and reagents}

Human umbilical vein endothelial cells (HUVECs) (ScienCell Research Laboratories, Carlsbad, CA, USA) were grown in endothelial cell medium supplemented with $5 \%$ foetal bovine serum (FBS), 100 units $/ \mathrm{mL}$ penicillin, and $100 \mu \mathrm{g} / \mathrm{mL}$ streptomycin. THP1 cells were obtained from the FuDan IBS Cell Center (Shanghai, China) and maintained in RPMI medium 1640 supplemented with $10 \%$ (vol/vol) FBS and 1\% penicillin-streptomycin. Trizol and CELLTRACE Violet were obtained from Invitrogen (Carlsbad, CA, USA). iScript gDNA Clear cDNA Synthesis Kit and SsoFast EvaGreen Supermix were obtained from Bio-RAD. Antibodies against p-YAP (Ser-127), YAP, TAZ, ICAM1, VCAM1, AMPK, p-AMPK, LATS1, p-LATS1 (Thr 1079), and $\beta$-actin used for western blotting were all purchased from Cell Signaling Technology (CST, Danvers, MA, USA). Antibodies against YAP and p-YAP used for immunofluorescence staining were purchased from CST and antibodies against YAP/TAZ used for immunofluorescence staining were purchased from Abcam (Cambridge, UK). A directly conjugated Alexa Fluor 488-CD31 antibody was purchased from Biolegend (San Diego, CA). An Annexin V-FITC Apoptosis Detection Kit was purchased from Becton-Dickinson (Franklin Lakes, NJ, USA). Small interfering RNAs against YAP
(siYAP) and AMPK $\alpha 1$ (siAMPK $\alpha 1$ ), as well as the negative control molecule (siNC), were purchased from RiboBio (Guangzhou, China). Methotrexate (in vitro) and atorvastatin were purchased from Aladdin (Shanghai, China). MTX (in vivo) was purchased from Pfizer (Bentley WA, Australia).

\section{Cell culture and treatment}

HUVECs were cultured in endothelial cell medium supplemented with $5 \% \mathrm{FBS}$ at $37{ }^{\circ} \mathrm{C}$ in a $5 \% \mathrm{CO}_{2}$ humidified atmosphere and passaged every $2-3$ days. Cells within seven passages were used for the in vitro study. For MTX treatment, before HUVECs were exposed to shear stress for the indicated durations $(0,1,10$, and $24 \mathrm{~h})$, they were preincubated with MTX $(100 \mathrm{nM})$ for $48 \mathrm{~h}$ [17]; the same procedure was used for atorvastatin $(1 \mu \mathrm{M})$ [18].

\section{Shear stress experiments}

A parallel-plate flow system was used to apply shear stress to HUVECs cultured in flow channels following previously established methods [19]. HUVECs were seeded onto fibronectin-coated glass slides and grown. The static (STA) treatment, as a control, unidirectional shear stress (USS), and DF were applied to HUVECs for 1,10 , or $24 \mathrm{~h}$ with a shear stress of $12 \mathrm{dyn} / \mathrm{cm}^{2}$ and $0 \pm 4$ $\mathrm{dyn} / \mathrm{cm}^{2}$.

\section{Western blotting}

Western blotting was used to detect the expression of target proteins in HUVECs. Briefly, equal amounts of total cell lysates were blotted onto a PVDF membrane and incubated with primary antibodies against YAP (1:1000), p-YAP (1:1000; Ser 127), TAZ (1:1000), ICAM-1 (1:1000), VCAM-1 (1:1000), p-AMPK (1:1000), AMPK (1:1000), and $\beta$-actin $(1: 1000)$ overnight at $4{ }^{\circ} \mathrm{C}$. Membranes were washed three times and then incubated with peroxidase-conjugated secondary antibody (1:5000) for $1 \mathrm{~h}$ at $37{ }^{\circ} \mathrm{C}$. Immunoreactive bands were detected by electrochemiluminescence (ECL) and exposure to X-ray film. Protein levels were quantified using scanning densitometry (ImageJ, National Institutes of Health, Bethesda, MD, USA). All data were obtained from three independent experiments.

\section{Monocyte adhesion assay}

THP1 monocytes were maintained in RPMI medium 1640 containing $10 \%(\mathrm{vol} / \mathrm{vol})$ FBS and $0.1 \%$ penicillin-streptomycin. THP-1 monocytes were labelled with CellTrace Violet and resuspended at $1 \times 10^{6}$ cells $/ \mathrm{mL}$ in 1640 medium. The adhesion assay was performed by adding the labelled THP-1 cells to HUVECs for $1 \mathrm{~h}$ at room temperature. After removing the unbound cells by 
two washes with 1640 medium, the THP-1 cells attached to HUVECs were fixed with $4 \%$ (wt/vol) PFA for $10 \mathrm{~min}$, and adhered THP1 cells were measured using a fluorescence microscope at $50 \times$ magnification.

\section{Immunofluorescence}

For in vitro experiments, HUVECs subjected to shear stress for $10 \mathrm{~h}$ were fixed with $4 \%$ (wt/vol) PFA for $10 \mathrm{~min}$. The cells were permeabilised with $0.3 \%$ triton $\mathrm{X}-100$ and blocked with 5\% (wt/vol) BSA in PBS for $30 \mathrm{~min}$, which was followed by incubation with the primary antibody against YAP, p-YAP $(1: 100)$ at $4{ }^{\circ} \mathrm{C}$ overnight. For arterial samples, frozen sections were embedded with optimal cutting temperature (OCT) compound, permeabilized, blocked, and incubated with primary antibodies against YAP/TAZ (1:100) at $4{ }^{\circ} \mathrm{C}$ overnight. After the incubation with primary antibodies, goat anti-rabbit IgG antibodies were used (1:100) as the secondary antibodies. The endothelial areas were identified in the anti-CD31 stained image, with anti-CD31 used as an endothelial marker. Nuclei were counterstained with DAPI. Images were obtained under a confocal microscope.

\section{Apoptosis assays}

The FITC Annexin V Apoptosis Detection Kit I was used to quantitatively determine the percentage of HUVECs undergoing apoptosis according to the manufacturer's instructions. After $10 \mathrm{~h}$ of shear stress, cells were harvested with trypsin (Beyotime Biotechnology, Haimen, China) and washed twice with cold PBS. Cells were washed twice with cold PBS and resuspended in $100 \mathrm{~mL}$ of binding buffer at a concentration of $1 \times 10^{6}$ cells $/ \mathrm{mL}$. Then, $5 \mu \mathrm{L}$ of FITC Annexin V and $5 \mu \mathrm{L}$ of propidium iodide were added to the cell suspension, followed by incubation at $25^{\circ} \mathrm{C}$ for $15 \mathrm{~min}$ in the dark with gentle vortexing for double staining. Then, $400 \mu \mathrm{L}$ of $1 \times$ Binding Buffer was added to each sample and immediately analysed by fluorescenceactivated cell sorting (FACS) using a flow cytometer (BD Biosciences, San Diego, CA, USA). The data were analysed using FlowJo (FlowJo LLC, Ashland, OR, USA).

\section{siRNA transfection}

HUVECs were transfected with serum-free media containing $50 \mu \mathrm{M}$ siRNA using the X-treme siRNA Transfection Reagent (RiboBio, Guangzhou, China) in accordance with the manufacturer's instructions. The transfection reagent was incubated for $15 \mathrm{~min}$ at room temperature before it was added to HUVECs and incubated for an additional $24 \mathrm{~h}$. After another $48 \mathrm{~h}$ with MTX, the HUVECs on slides were used for the shear stress experiment. The following targeted siRNAs were synthesised by RiboBio: siRNA-AMPKa1 (GATCCATCATATAGTTCA A) and siRNA-YAP1 (CCACCAAGCTAGATAAAGA).

\section{qRT-PCR analyses}

Total RNA was isolated from HUVECs using TRIzol according to the manufacturer's instructions. First-strand cDNA was generated from $1 \mu \mathrm{g}$ of RNA using the iScript gDNA Clear cDNA Synthesis Kit. qRT-PCR experiments were performed using the SsoFast EvaGreen Supermix. Glyceraldehyde 3-phosphate dehydrogenase (GAPDH) was used as a standard control for normalisation. Each cDNA sample was run in triplicate. qPCR data were analysed using the $2^{-\triangle \Delta C T}$ cycle threshold method. Table 1 presents all primers used for qRT-PCR.

\section{Animals}

Male C57BL/6 mice weighing at least $18 \mathrm{~g}$ were purchased from the Second Affiliated Hospital of the Harbin Medical University Laboratory Animal Centre and fed a Western-type diet. All animal care was conducted in accordance with the "Principles of Animal Care" (Ethical and Animal Welfare Committee of Heilongiang Province, China) and were approved by the ethics review board of Harbin Medical University. Briefly, after anaesthetisation with isoflurane, DF was altered by cast placement in the left common carotid artery, as previously described [20]. Animals were randomly allocated to two groups; one group was treated with MTX at $1 \mathrm{mg} /$ $\mathrm{kg} /$ week [21] and the other group received an equal volume of $0.9 \%$ saline by weekly intraperitoneal injections. The treatments began on the day of cast placement. Four weeks later, all animals were sacrificed. The carotid artery was embedded in OCT compound and 7- $\mu \mathrm{m}$ cryosections were prepared for haematoxylin-eosin (H\&E) staining, Masson staining, and immunofluorescence.

\section{Statistical analysis}

Data are expressed as mean $\pm \mathrm{SD}$. Student's $t$-tests were used to evaluate differences between two groups and a one-way ANOVA with Tukey's post hoc test was used

\section{Table 1 Primers for qRT-PCR}

\begin{tabular}{lll}
\hline Gene & Forward & Reverse \\
\hline AMPK-a & TGATGTTGTAGTGACACCATTTAC & GAAGATGAGGGAAAGAATTAAGGG \\
CTGF & ACCGACTGGAAGACACGTTTG & CCAGGTCAGCTTCGCAAGG \\
CYR-61 & TGAAGCGGCTCCCTGTTT & CGGGTTCTTTCACAAGGCG \\
VCAM1 & CAGTAAGGCAGGCTGTAAAAGA & TGGAGCTGGTAGACCCTCG \\
ICAM1 & TTGGGCATAGAGACCCCGTT & GCACATTGCTCAGTTCATACACC \\
IL-6 & CTCAATATTAGAGTCTCAACCCCCA & GAGAAGGCAACTGGACCGAA \\
IL-8 & CCACCGGAGCACTCCATAAG & GATGGTTCCTTCCGGTGGTT \\
YAP & GCTACAGTGTCCCTCGAACC & CCGGTGCATGTGTCTCCTTA \\
TAZ & ATCCCCAACAGACCCGTTTC & GAACGCAGGCTTGCAGAAAA \\
GAPDH & ACGGATTTGGTCGTATTGGGC & TTGACGGTGCCATGGAATTTG \\
\hline
\end{tabular}


for multiple groups. GraphPad Prism version 7.0 was used for analyses and $\mathrm{p}<0.05$ was considered statistically significant.

\section{Results}

Haemodynamic regulation of the activation and nuclear localisation of YAP/TAZ in HUVECs

To investigate the roles of YAP/TAZ in HUVECs under different shear forces, cultured HUVECs were exposed to USS, DF, or STA for $10 \mathrm{~h}$. We measured the protein expression levels of p-YAP (Ser127), total-YAP/TAZ, and adhesion molecules by western blotting. Our results demonstrated that DF leads to a significant decrease in p-YAP (Ser127) (Fig. 1a, b) and marked increases in ICAM1, VCAM1, and TAZ expression (Fig. 1a). IF staining showed that DF results in YAP/TAZ nuclear localisation, whereas increased YAP/TAZ cytoplasmic retention was observed in HUVECs subjected to USS. In addition, the levels of YAP phosphorylation were also examined by IF; we found that DF markedly decreased p-YAP (Fig. 1c), consistent with the western blotting results (Fig. 1a).
DF promoted the transactivation activity of YAP/TAZ and significantly increased the expression of the YAP/ TAZ target genes CYR61 and CTGF (Fig. 2b). In contrast, USS enhanced p-YAP (Figs. 1a, 2c) and reduced YAP/TAZ target gene expression (Fig. 2b). Moreover, biomechanical stretch mediates the ability of HUVECs to recruit monocytes. We used a cell adhesion assay and found an increased number of THP1 monocytes attached to HUVECs under DF (Fig. 1d), which was accompanied by the up-regulation of adhesion molecules (Fig. 1a). These results indicated that DF leads to YAP dephosphorylation and activation, whereas USS results in YAP phosphorylation and inactivation.

\section{MTX phosphorylates AMPK and alleviates DF-induced proinflammatory cytokine expression in HUVECs}

HUVECs were treated with MTX $(0-100 \mathrm{nM})$ and p-AMPK levels were quantified after $48 \mathrm{~h}$ by western blotting. After treatment with MTX for 48 h, p-AMPK in HUVECs cultured in static conditions increased in a dose-dependent manner, with a maximum level at

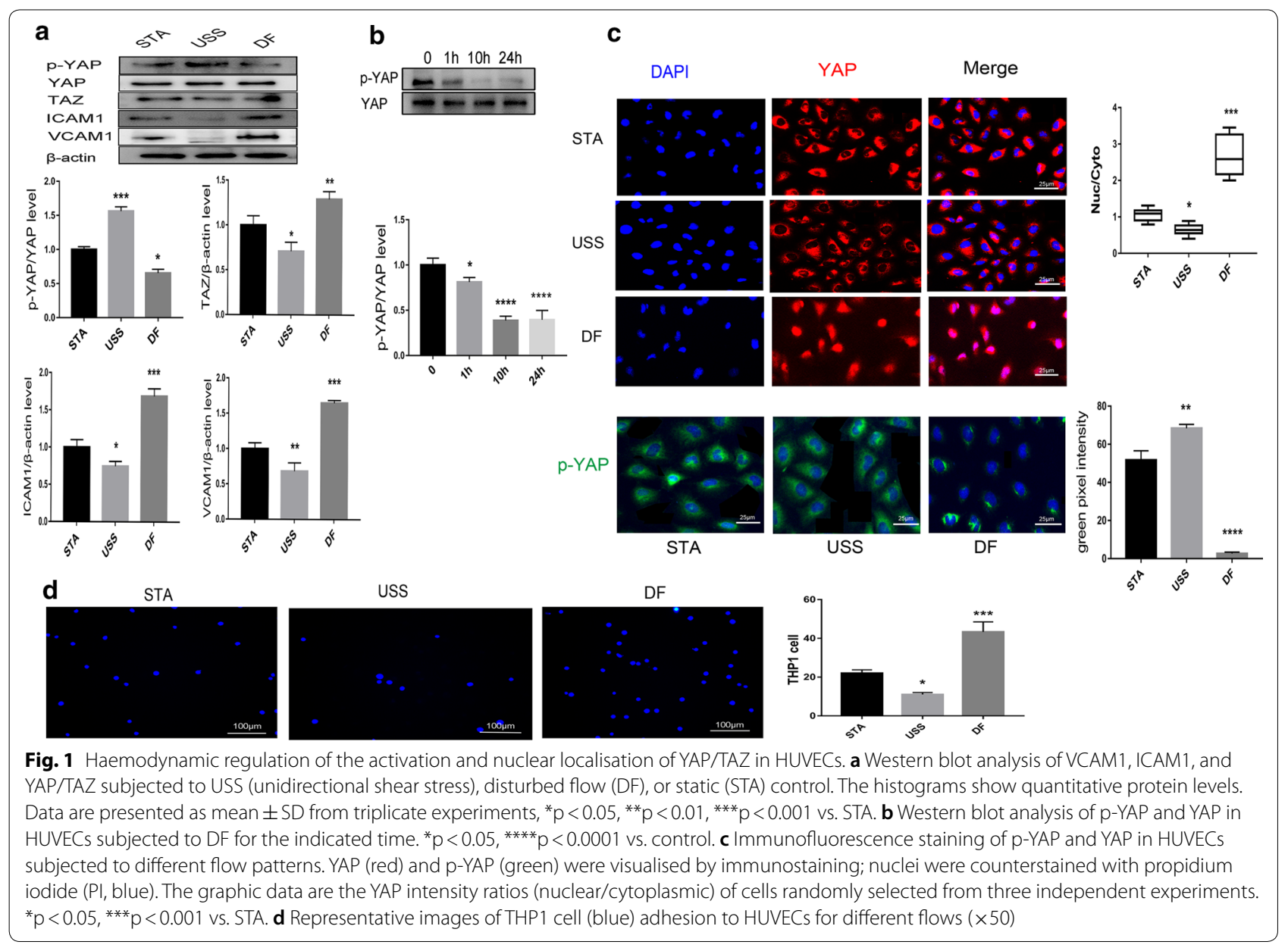


a
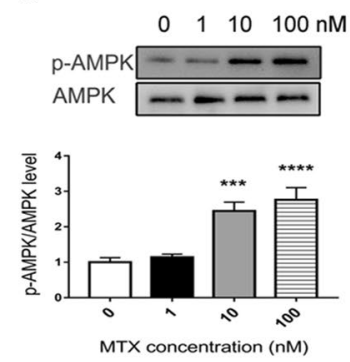

c

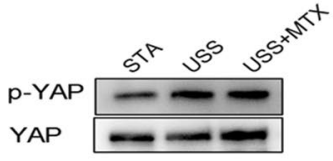

b
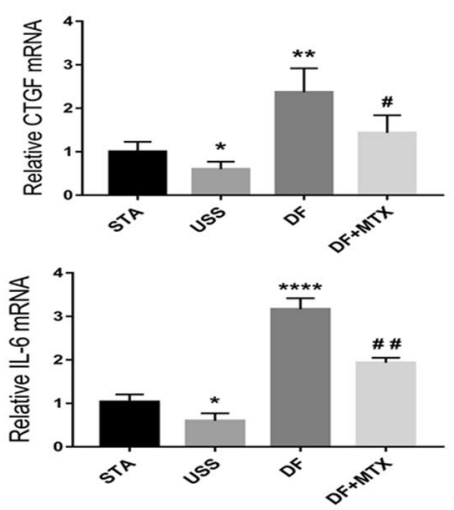
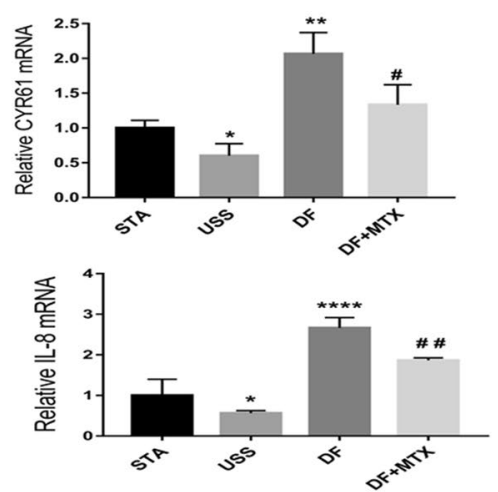

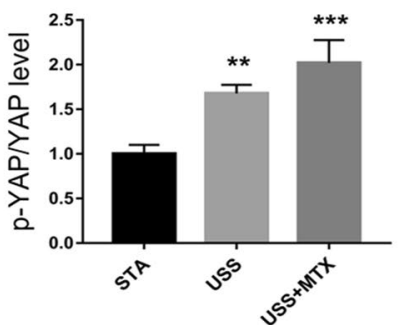

d
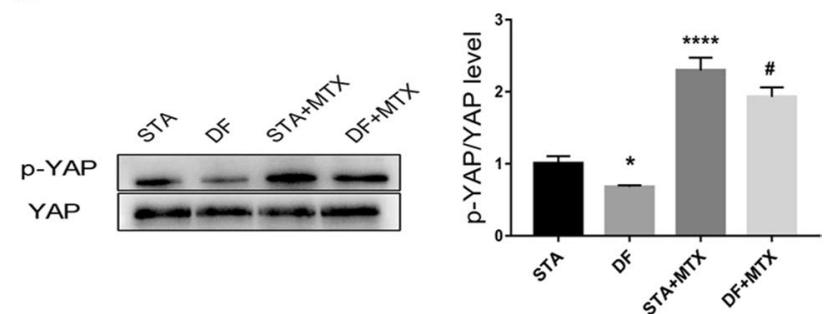

Fig. 2 MTX treatment increases the level of P-YAP and alleviates the expression of YAP target genes and inflammatory factors. a HUVECs were treated with MTX (0-100 nM) and p-AMPK was quantified after $48 \mathrm{~h}$ by a western blot analysis. ${ }^{* * *} \mathrm{p}<0.001,{ }^{* * *} \mathrm{p}<0.0001 \mathrm{vs}$. Control. b qRT-PCR analysis of YAP/TAZ target genes and inflammatory factors for different flows. Results are presented as mean \pm SD from three independent experiments from each group. ${ }^{*} p<0.05$, ${ }^{* *} p<0.01,{ }^{* * *} p<0.0001$ vs. STA (static), and ${ }^{\#} p<0.05$, ${ }^{\# \#} p<0.01$ vs. DF. c HUVECs were treated with or without MTX (100 nM) under USS, and p-YAP (Ser127) was quantified by western blot analysis. ${ }^{* *} \mathrm{p}<0.01,{ }^{* * *} \mathrm{p}<0.001$ vs. STA. d HUVECs were treated with or without MTX under STA or DF, and p-YAP (Ser127) was quantified by western blot analysis. ${ }^{*} p<0.05$, ${ }^{* * * *} p<0.0001$ vs. STA; ${ }^{*} p<0.05$ vs. STA + MTX

$100 \mathrm{nM}$ (Fig. 2a). The concentration was consistent with conventional low-dose therapeutic dosing for patient plasma [22].

To establish whether MTX alters proinflammatory cytokine expression under haemodynamic forces, qRTPCR was used to analyse levels of proinflammatory cytokine genes and YAP/TAZ target genes. Indeed, we observed higher levels of $I L-6, I L-8, C Y R 61$, and CTGF under DF rather than USS in HUVECs, and MTX treatment significantly suppressed their expression (Fig. 2b).

To determine whether the low-dose MTX induced changes downstream of AMPK, we examined the protein expression levels of p-YAP (Ser127) under different flow conditions. The results demonstrated that DF, but not USS, led to a significant decrease in YAP phosphorylation (Fig. 2c, d). However, when we compared the effects of MTX treatment vs. control under USS, we did not observe a significant change in YAP phosphorylation (Fig. 2c). MTX treatment led to a marked increase in $\mathrm{p}$-YAP expression under DF or STA, with the latter leading to higher $\mathrm{p}$-YAP expression after MTX treatment (Fig. 2d).
MTX inhibits DF-induced YAP/TAZ activation and exerts atheroprotective effects, whereas silencing AMPKa reverses these effects

We next explored whether MTX mediates YAP/TAZ inactivation in HUVECs under biomechanical stretch by immunofluorescence and western blotting. A western blot analysis revealed that MTX significantly upregulated the expression of $\mathrm{p}$-YAP and $\mathrm{p}$-AMPK and markedly decreased the levels of ICAM1 and VCAM1 under DF (Fig. 3a). We also observed that total YAP was constitutively expressed at high levels in the cytoplasm and that monocyte adhesion was reduced after MTX (100 nM) treatment under DF (Fig. 3b, c). These data suggest that MTX mediates YAP inhibition and reduces monocyte-HUVEC interactions, contributing, at least in part, to anti-atherogenic effects.

Because YAP can be phosphorylated by AMPK [23], we further explored the role of AMPK in this MTXinduced protective effect and determined whether MTX-mediated YAP phosphorylation is AMPKdependent. AMPK $\alpha$ depletion by siRNA (Fig. 3d) promoted YAP nuclear translocation and nearly abolished MTX-mediated YAP phosphorylation (Fig. 3a) and 


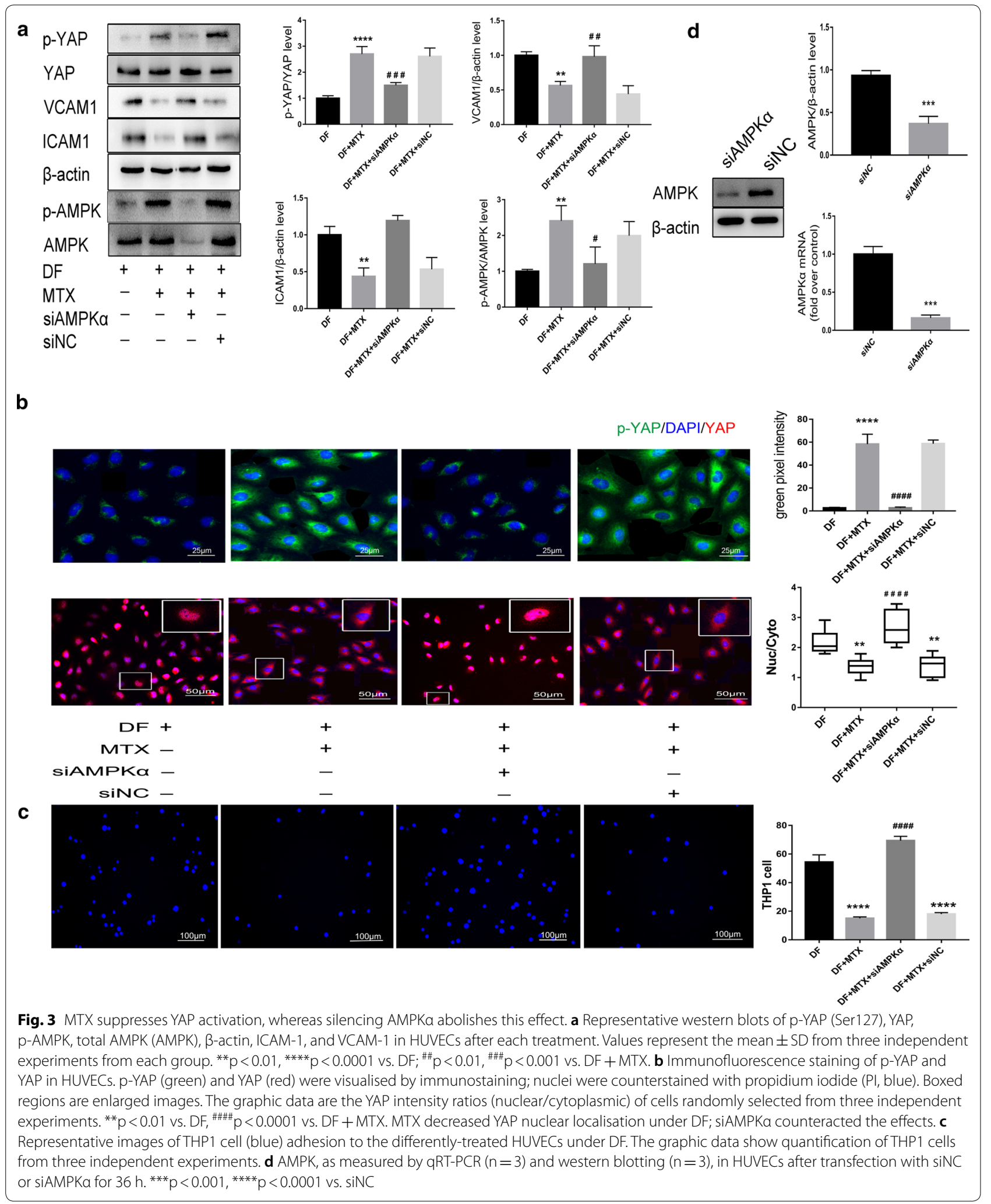


p-YAP cytoplasmic localisation (Fig. 3b), indicating a critical role of AMPK in mediating MTX-induced YAP inactivation. Following AMPK knockdown in HUVECs, both the levels of monocyte adhesion proteins and the augmentation of monocyte adhesion were significantly increased under DF (Fig. 3a, c).

\section{Silencing YAP reduces adhesion molecule expression under DF}

To further confirm the association between YAP and DF-induced proinflammatory responses, siRNAs against YAP were used to examine the interactions among YAP, adhesion molecules, and AMPK. Western blotting results demonstrated that knocking down YAP significantly inhibited the expression of ICAM1 and VCAM1 but did not alter p-AMPK protein levels under DF (Fig. 4a, b), indicating that AMPK acts upstream of YAP, consistent with previous results [14], and that YAP depletion might contribute to atherogenesis.

\section{Effects of MTX on DF-induced apoptosis and YAP phosphorylation}

Impaired endothelial function comprises an early stage of atherosclerosis. In this study, flow cytometry was used to assay cell apoptosis under DF. Compared to that with
STA, the application of DF, but not USS, significantly increased the total apoptosis rate. However, treatment with MTX did not reduce apoptosis (Fig. 5a, b).

Using western blot analyses, we detected comparable levels of p-YAP expression in HUVECs treated with MTX and the widely-used atherosclerotic drug atorvastatin under DF. Our results demonstrated that the exposure of cells to DF in the presence of MTX $(100 \mathrm{nM})$ or atorvastatin $(1 \mu \mathrm{M})$ resulted in the hyperphosphorylation of YAP. There was no statistically significant difference in the level of p-YAP expression between MTX and statin treatments (Fig. 5c).

\section{MTX treatment decreases DF-induced plaque formation in vivo}

To identify the potential anti-arteriosclerosis effects of low-dose MTX in vivo, a cast was placed around the left common carotid artery to induce DF. After exposure to a Western diet for 4 weeks, apparent atherosclerotic lesions were induced in the DF region (Fig. 6a). YAP/TAZ staining was remarkable in the intima, the media layer, and the intimal hyperplastic plaque of the atherosclerotic section (Fig. 6b). In contrast, treating mice with MTX resulted in a significant reduction

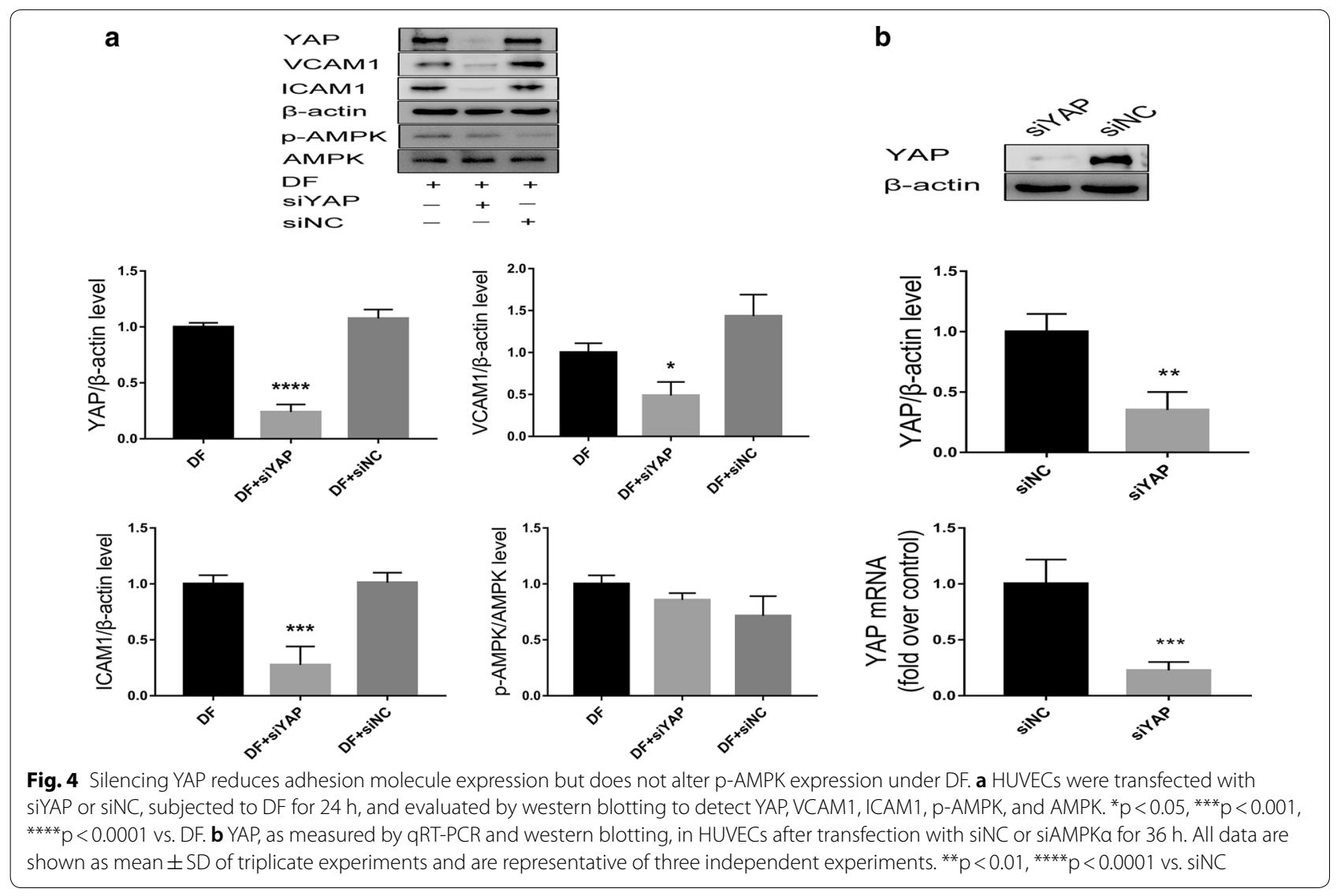



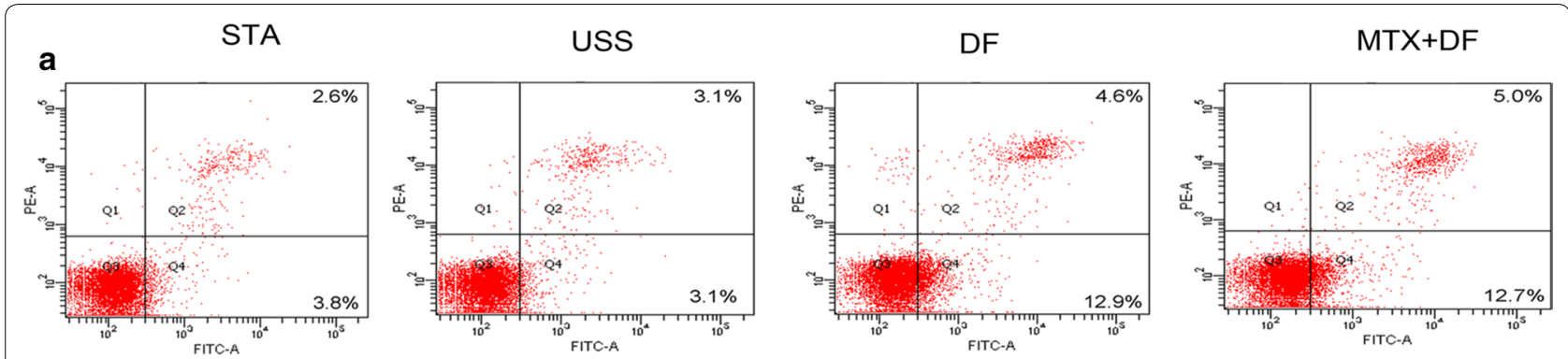

b

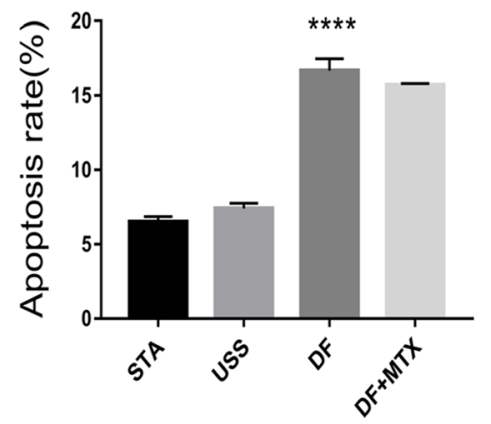

C
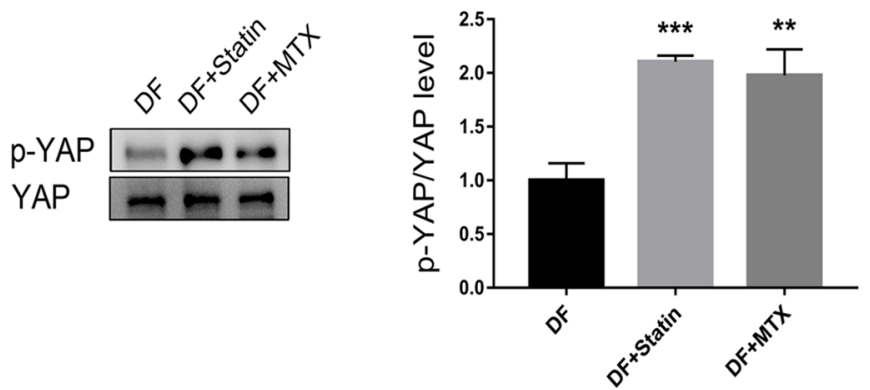

Fig. 5 MTX has no effect on DF-induced HUVEC apoptosis and atorvastatin promotes YAP phosphorylation. a HUVECs were incubated with MTX for $48 \mathrm{~h}$ and subjected to biomechanical stretch for another $10 \mathrm{~h}$. Apoptosis in HUVECs is shown as flow cytometry dot plots. b As quantified by flow cytometry, DF increased the total apoptosis rate compared to that under static (STA) conditions. However, treatment with MTX did not reduce apoptosis. The percentage of apoptotic cells is presented as the mean \pm SD from triplicate experiments. ${ }^{* * *} p<0.0001$ vs. STA. c Western blotting results (left panel) and quantitative data (right panel) for the protein levels of p-YAP are presented. Values are presented as mean $\pm S D$ from three independent experiments. ${ }^{* *} p<0.01,{ }^{* * *} p<0.001 \mathrm{vs}$. DF

in atherosclerotic plaque development (Fig. 6a, b) and relative YAP/TAZ expression (Fig. 6c). Moreover, the lumen in the DF region was larger than that with $0.9 \%$ saline treatment (Fig. 6a).

\section{Discussion}

Atherosclerosis is considered an inflammatory disease $[24,25]$. Recently, MTX has attracted increasing attention due to its vasculoprotective and anti-inflammatory effects. In this study, we show that the MTX-YAP/TAZ pathway has a profound effect on the development of DF-induced atherosclerosis (Fig. 7). Our results demonstrated that DF leads to YAP activation, a decrease in YAP phosphorylation (Fig. 1a, b) and a marked increase in total YAP expression (Fig. 6c), in accordance with previous results $[6,8]$, and that the atheroprotective effect of MTX is mediated by the inhibition of YAP activity and up-regulation of p-YAP (Ser127). MTX markedly decreased pro-inflammatory factor secretion and monocyte adhesion under DF (Figs. 2b, 3a, c), which in turn contributed to atherogenesis. These beneficial effects were reversed upon silencing endogenous AMPK $\alpha$ (Fig. 3a, b). Furthermore, MTX-treated mice not only exhibited a significant reduction in plaque development but also lower YAP/TAZ expression under DF (Fig. 6a, c). Taken together, these results indicate that YAP/TAZ may serve as important regulators of atherosclerosis and that MTX might have atheroprotective effects via the inactivation of YAP/TAZ. Accordingly, MTX/AMPK/YAP represents a promising therapeutic target that reduces the expression of atherogenic factors in HUVECs.

A crucial event in atherosclerosis is endothelial dysfunction resulting from haemodynamic forces [26]. HUVECs and human coronary artery endothelial cells, which express abundant YAP, are constantly exposed to mechanical forces generated by the blood flow [27]. YAP/ TAZ, which are key factors in the pathophysiology of the cardiovascular system [28], sense a highly diverse range of mechanical cues and translate these cues into specific biochemical signals. YAP located in the nucleus under DF promotes endothelial cell ICAM1 and VCAM1 expression for monocyte adherence, which is correlated with key pathogenic events in atherosclerosis such as endothelial thickening and the recruitment of monocytes, which eventually turn into plaques [7]. Therefore, both YAP depletion and inhibition might be effective strategies to retard atherogenesis. Our results showed that MTX can suppress YAP activation and reduce the levels of 
a

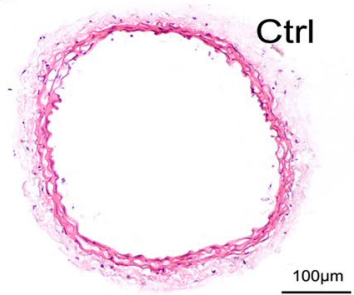

b

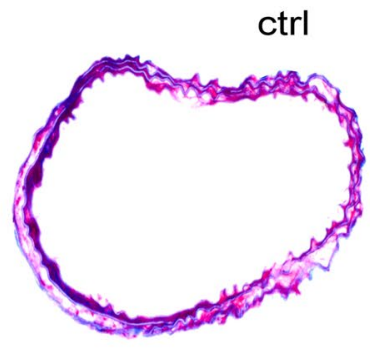

$\underline{100 \mu \mathrm{m}}$

C
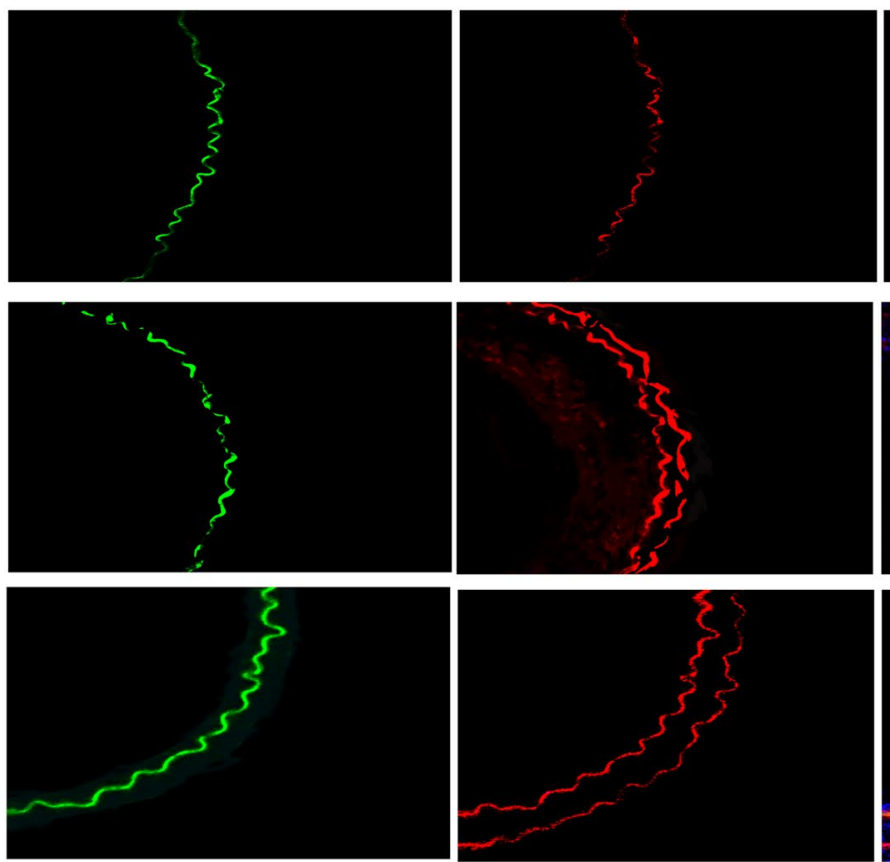

DF
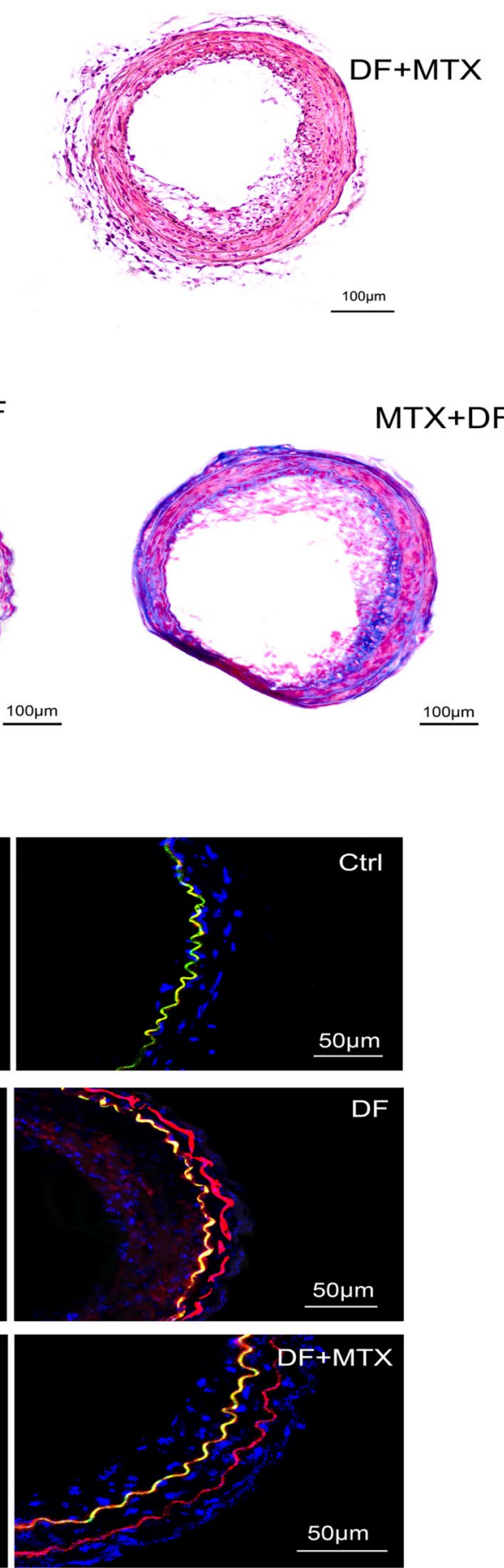

EC/YAP/TAZ/DAPI

Fig. 6 MTX-treated animals present with a lower plaque burden and lower relative YAP/TAZ expression levels in DF regions. $\mathbf{a}$, $\mathbf{b}$ Representative H\&E and Masson-stained normal left carotid artery $(n=3)$ sections from the upstream region of the cast $(0.5 \mathrm{~mm}$ proximal from the cast) where DF was induced after $0.9 \%$ saline treatment for 4 weeks $(n=7)$ and sections after treatment with MTX $(n=8)$. c Representative immunofluorescence image of the normal left carotid artery treated with $0.9 \%$ saline or MTX. Sections were stained with YAP/TAZ (red) and DAPI (Blue). Sections are stained with anti-CD31 as an endothelial marker (green). MTX-treated animals showed lower relative YAP/TAZ expression levels in DF regions 


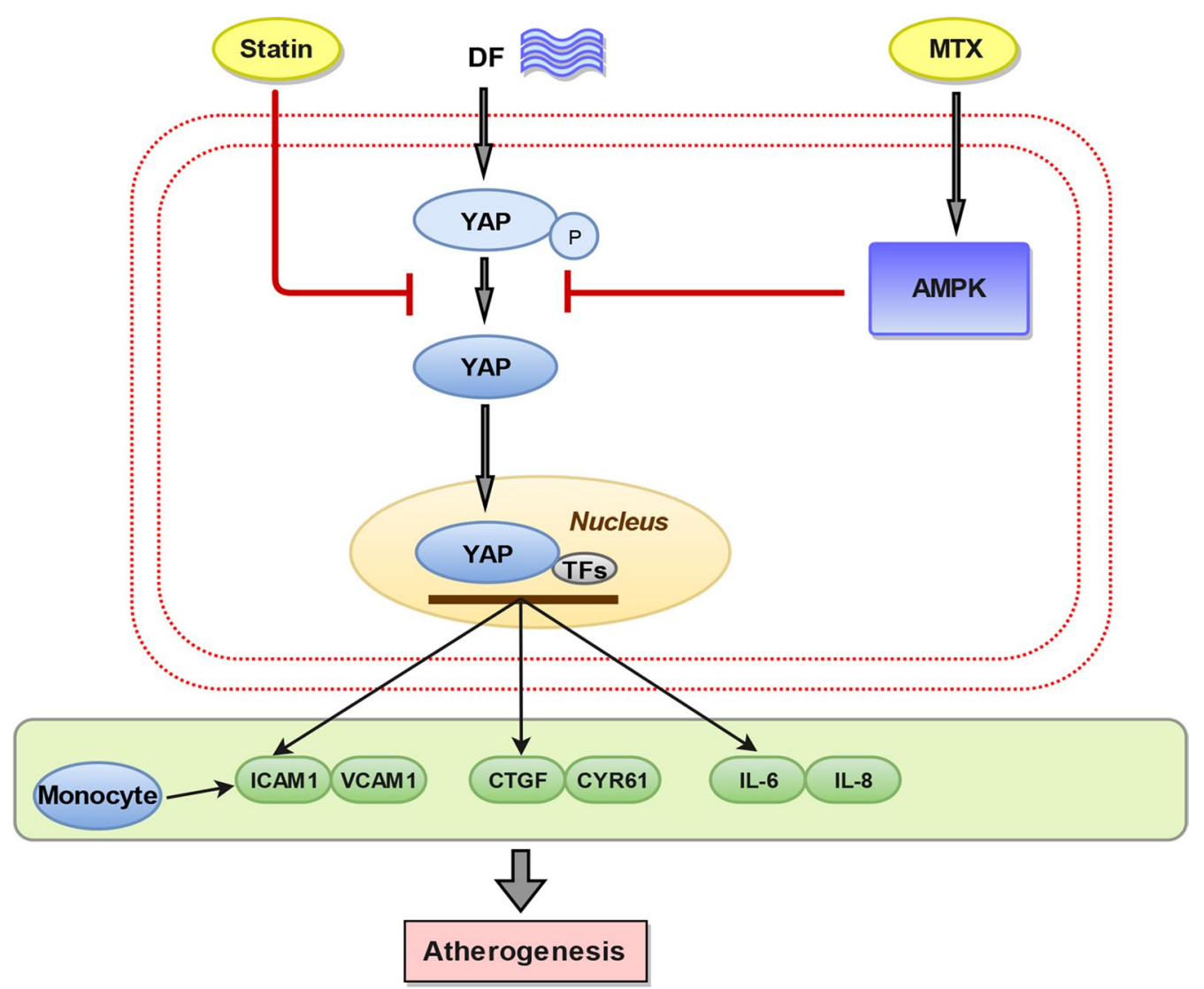

Fig. 7 Schematic diagram of the possible mechanism underlying methotrexate (MTX)-mediated protection of HUVECs against disturbed flow (DF) injury via the activation of AMPK-YAP/TAZ signalling

inflammatory factors and adhesion molecules, (Figs. 2b, $3 a, c)$, indicating that it might indeed be effective for the treatment of atherosclerosis. Both MTX and USS can cause an increase in $\mathrm{p}$-YAP, but we did not observe a significant difference in $\mathrm{p}$-YAP between USS and MTF + USS (Fig. 2c); we speculate that this is because it had already reached its maximum. Statins, which are widely used drugs that lower cellular cholesterol levels, have the ability to repress YAP activity and prevent YAPmediated transcription [29]. Indeed, we observed that HUVECs treated with atorvastatin or MTX showed similar p-YAP protein levels. However, the exact molecular mechanism underlying these results should be evaluated in further studies.

Anti-inflammatory therapeutic strategies hold great potential for halting the progression and inducing the regression of atherosclerosis. MTX-loaded hybrid nanoconstructs target vascular lesions and inhibit atherosclerosis progression in $\mathrm{ApoE}^{-/-}$mice [30]. MTX has been successfully used for the treatment of many immune or inflammatory diseases [31]. These positive effects of MTX on cardiovascular disease have also caught the attention of cardiologists. We previously found that MTX can reduce in-stent neoatherosclerosis in a rabbit model and that neoatherosclerosis frequently occurs at both edges of a stent affected by unidirectional shear flow [32], suggesting that local fluid shear stress is involved in in-stent neoatherosclerosis. Therefore, a better understanding of the effects of MTX on atherosclerosis under shear stress has clinical significance, and our experiment provides preclinical evidence that YAP/TAZ are potential therapeutic targets for in-stent neoatherosclerosis. However, the mechanism underlying the atheroprotective effect of MTX has not been reported. With respect to metabolic characteristics, MTX first inhibits AICAR transformylase and then results in AICAR accumulation and AMPK phosphorylation [17]. AMPK activity is associated with anti-inflammatory effects and exerts multiple protective effects during atherosclerosis [33, 34]. Several lines of evidence suggest that AMPK can inhibit YAP directly by phosphorylation of YAP and activation of the Lats kinase indirectly, resulting in YAP inactivation and 
causing their cytoplasmic retention and degradation [35]. Our data show that MTX treatment increased p-AMPK and p-YAP (Ser127) expression. Consistent with these findings, it is plausible that the AMPK pathway is responsible for MTX-mediated YAP (Ser127) phosphorylation based on our results indicating that the beneficial effects of MTX on DF-induced HUVECs are abolished by the knockdown of endogenous AMPK $\alpha$. The protective effects of MTX were mediated, at least in part, by the suppression of AMPK activation. These results support the use of MTX for the treatment of atherosclerosis.

\section{Conclusion}

From a therapeutic perspective, these findings provide insight into the mechanism through which MTX confers atheroprotection and might facilitate the development of new therapeutic approaches to limit atherosclerosis.

\section{Abbreviations \\ YAP/TAZ: yes-associated protein and transcriptional co-activator with PDZ-binding motif; MTX: methotrexate; AMPK: adenosine monophosphate activated protein kinase; DF: disturbed flow.}

\section{Acknowledgements}

Not applicable.

\section{Authors' contributions}

$\mathrm{DL}$ and $\mathrm{HL}$ designed the study; $\mathrm{DL}, \mathrm{QL}, \mathrm{YS}$ and SH carried out experiments. Data analysis was carried out by $\mathrm{DL}, \mathrm{BH}, \mathrm{LZ}, \mathrm{MY}, \mathrm{BY}$ and $\mathrm{XW}$. The mice were managed by RZ, GW and HN. The paper was written by DL, HL, WD and XH. All authors read and approved the final manuscript.

\section{Funding}

This work was supported by the National Natural Science Foundation of China (Grant Nos. 81801803 and 81671794) and the Postdoctoral Sustentation Fund of Harbin Medical University (Grant Nos. 2018M640310, 2018M641870 and $\mathrm{LBH}-\mathrm{Z18141).}$

\section{Availability of data and materials}

All relevant data and materials are included in this published article.

\section{Ethics approval and consent to participate}

All animal care was conducted in accordance with the "Principles of Animal Care" (Ethical and Animal Welfare Committee of Heilongjiang Province, China) and were approved by the ethics review board of Harbin Medical University.

\section{Consent for publication}

All co-authors have read the manuscript and approved its submission to Journal of Translational Medicine.

\section{Competing interests}

The authors declare that they have no competing interests.

\begin{abstract}
Author details
${ }^{1}$ Division Department of Cardiology Organization, The Second Affiliated Hospital of Harbin Medical University, Harbin, China. ${ }^{2}$ Key Laboratories of the Education Ministry for Myocardial Ischemia Mechanisms and Treatment, Harbin, China. ${ }^{3}$ Division of Cardiovascular Surgery, The Second Affiliated Hospital of Harbin Medical University, Harbin, China.
\end{abstract}

Received: 26 June 2019 Accepted: 9 November 2019 Published online: 15 November 2019

\section{References}

1. Roth GA, Forouzanfar MH, Moran AE, Barber R, Nguyen G, Feigin VL, Naghavi M, Mensah GA, Murray CJ. Demographic and epidemiologic drivers of global cardiovascular mortality. N Engl J Med. 2015;372:1333-41.

2. Herrington W, Lacey B, Sherliker P, Armitage J, Lewington S. Epidemiology of atherosclerosis and the potential to reduce the global burden of atherothrombotic disease. Circ Res. 2016;118:535-46.

3. Kim CW, Pokutta-Paskaleva A, Kumar S, Timmins LH, Morris AD, Kang DW, Dalal S, Chadid T, Kuo KM, Raykin J, et al. Disturbed flow promotes arterial stiffening through thrombospondin-1. Circulation. 2017;136:1217-32.

4. Wentzel JJ, Schuurbiers JC, Gonzalo Lopez N, Gijsen FJ, van der Giessen AG, Groen HC, Dijkstra J, Garcia-Garcia HM, Serruys PW. In vivo assessment of the relationship between shear stress and necrotic core in early and advanced coronary artery disease. Eurolntervention. 2013;9:989-95 (discussion 995).

5. Zhou J, Li YS, Chien S. Shear stress-initiated signaling and its regulation of endothelial function. Arterioscler Thromb Vasc Biol. 2014;34:2191-8.

6. Wang KC, Yeh YT, Nguyen P, Limqueco E, Lopez J, Thorossian S, Guan KL, Li YJ, Chien S. Flow-dependent YAP/TAZ activities regulate endothelial phenotypes and atherosclerosis. Proc Natl Acad Sci USA. 2016;113:11525-30.

7. Panciera T, Azzolin L, Cordenonsi M, Piccolo S. Mechanobiology of YAP and TAZ in physiology and disease. Nat Rev Mol Cell Biol. 2017;18:758-70.

8. Wang L, Luo JY, Li B, Tian XY, Chen LJ, Huang Y, Liu J, Deng D, Lau CW, Wan $\mathrm{S}$, et al. Integrin-YAP/TAZ-JNK cascade mediates atheroprotective effect of unidirectional shear flow. Nature. 2016;540:579-82.

9. Micha R, Imamura F, Wyler von Ballmoos M, Solomon DH, Hernan MA, Ridker PM, Mozaffarian D. Systematic review and meta-analysis of methotrexate use and risk of cardiovascular disease. Am J Cardiol. 2011;108:1362-70.

10. Choi HK, Hernan MA, Seeger JD, Robins JM, Wolfe F. Methotrexate and mortality in patients with rheumatoid arthritis: a prospective study. Lancet. 2002;359:1173-7.

11. Zhang R, Chen S, Zhang H, Liu Q, Xing J, Zhao Q, Wang Y, Yu B, Hou J. Effects of methotrexate in a rabbit model of in-stent neoatherosclerosis: an optical coherence tomography study. Sci Rep. 2016;6:33657.

12. Cronstein BN, Eberle MA, Gruber HE, Levin RI. Methotrexate inhibits neutrophil function by stimulating adenosine release from connective tissue cells. Proc Natl Acad Sci USA. 1991;88:2441-5.

13. Chan ES, Cronstein BN. Methotrexate-how does it really work? Nat Rev Rheumatol. 2010;6:175-8.

14. DeRan M, Yang J, Shen CH, Peters EC, Fitamant J, Chan P, Hsieh M, Zhu $\mathrm{S}$, Asara JM, Zheng B, et al. Energy stress regulates hippo-YAP signaling involving AMPK-mediated regulation of angiomotin-like 1 protein. Cell Rep. 2014;9:495-503.

15. Mo JS, Meng Z, Kim YC, Park HW, Hansen CG, Kim S, Lim DS, Guan KL. Cellular energy stress induces AMPK-mediated regulation of YAP and the Hippo pathway. Nat Cell Biol. 2015;17:500-10.

16. Wang W, Xiao ZD, Li X, Aziz KE, Gan B, Johnson RL, Chen J. AMPK modulates Hippo pathway activity to regulate energy homeostasis. Nat Cell Biol. 2015;17:490-9.

17. Thornton CC, Al-Rashed F, Calay D, Birdsey GM, Bauer A, Mylroie H, Morley BJ, Randi AM, Haskard DO, Boyle JJ, Mason JC. Methotrexate-mediated activation of an AMPK-CREB-dependent pathway: a novel mechanism for vascular protection in chronic systemic inflammation. Ann Rheum Dis. 2016;75:439-48.

18. Giordano A, Romano S, Monaco M, Sorrentino A, Corcione N, Di Pace AL, Ferraro P, Nappo G, Polimeno M, Romano MF. Differential effect of atorvastatin and tacrolimus on proliferation of vascular smooth muscle and endothelial cells. Am J Physiol Heart Circ Physiol. 2012;302:H135-42.

19. Wang KC, Nguyen P, Weiss A, Yeh YT, Chien HS, Lee A, Teng D, Subramaniam S, LiYS, Chien S. MicroRNA-23b regulates cyclin-dependent kinaseactivating kinase complex through cyclin $\mathrm{H}$ repression to modulate endothelial transcription and growth under flow. Arterioscler Thromb Vasc Biol. 2014;34:1437-45.

20. Cheng C, Tempel D, van Haperen R, de Boer HC, Segers D, Huisman M, van Zonneveld AJ, Leenen PJ, van der Steen A, Serruys PW, et al. Shear stress-induced changes in atherosclerotic plaque composition are modulated by chemokines. J Clin Investig. 2007;117:616-26. 
21. Refaat R, Salama M, Abdel Meguid E, El Sarha A, Gowayed M. Evaluation of the effect of losartan and methotrexate combined therapy in adjuvant-induced arthritis in rats. Eur J Pharmacol. 2013;698:421-8.

22. Combe B, Edno L, Lafforgue P, Bologna C, Bernard JC, Acquaviva P, Sany J, Bressolle F. Total and free methotrexate pharmacokinetics, with and without piroxicam, in rheumatoid arthritis patients. Br J Rheumatol. 1995:34:421-8

23. von Eyss B, Jaenicke LA, Kortlever RM, Royla N, Wiese KE, Letschert S, McDuffus LA, Sauer M, Rosenwald A, Evan Gl, et al. A MYC-driven change in mitochondrial dynamics limits YAP/TAZ function in mammary epithelial cells and breast cancer. Cancer Cell. 2015;28:743-57.

24. van Leuven SI, Franssen R, Kastelein JJ, Levi M, Stroes ES, Tak PP. Systemic inflammation as a risk factor for atherothrombosis. Rheumatology. 2008;47:3-7.

25. Goodson NJ, Symmons DP, Scott DG, Bunn D, Lunt M, Silman AJ. Baseline levels of C-reactive protein and prediction of death from cardiovascular disease in patients with inflammatory polyarthritis: a ten-year followup study of a primary care-based inception cohort. Arthritis Rheum. 2005;52:2293-9.

26. Cunningham KS, Gotlieb Al. The role of shear stress in the pathogenesis of atherosclerosis. Lab Investig. 2005;85:9-23.

27. Xu S, Koroleva M, Yin M, Jin ZG. Atheroprotective laminar flow inhibits Hippo pathway effector YAP in endothelial cells. Transl Res. 2016;176(18-28):e12.

28. Hahn C, Schwartz MA. Mechanotransduction in vascular physiology and atherogenesis. Nat Rev Mol Cell Biol. 2009;10:53-62.

29. Sorrentino G, Ruggeri N, Specchia V, Cordenonsi M, Mano M, Dupont S, Manfrin A, Ingallina E, Sommaggio R, Piazza S, et al. Metabolic control of YAP and TAZ by the mevalonate pathway. Nat Cell Biol. 2014;16:357-66.
30. Stigliano C, Ramirez MR, Singh JV, Aryal S, Key J, Blanco E, Decuzzi P. Methotraxate-loaded hybrid nanoconstructs target vascular lesions and inhibit atherosclerosis progression in $\mathrm{ApoE}(-/-)$ mice. Adv Healthc Mater. 2017;6:1201686. https://doi.org/10.1002/adhm.201601286.

31. Reiss AB, Carsons SE, Anwar K, Rao S, Edelman SD, Zhang H, Fernandez $\mathrm{P}$, Cronstein BN, Chan ES. Atheroprotective effects of methotrexate on reverse cholesterol transport proteins and foam cell transformation in human THP-1 monocyte/macrophages. Arthritis Rheum. 2008:58:3675-83.

32. Tian J, Ren X, Uemura S, Dauerman H, Prasad A, Toma C, Jia H, Abtahian F, Vergallo R, Hu S, et al. Spatial heterogeneity of neoatherosclerosis and its relationship with neovascularization and adjacent plaque characteristics: optical coherence tomography study. Am Heart J. 2014;167(884-892):e882.

33. Wang S, Zhang M, Liang B, Xu J, Xie Z, Liu C, Viollet B, Yan D, Zou MH. AMPKalpha2 deletion causes aberrant expression and activation of $\mathrm{NAD}(\mathrm{P}) \mathrm{H}$ oxidase and consequent endothelial dysfunction in vivo: role of 26S proteasomes. Circ Res. 2010;106:1117-28.

34. Ewart MA, Kennedy S. AMPK and vasculoprotection. Pharmacol Ther. 2011;131:242-53.

35. Choi HJ, Zhang H, Park H, Choi KS, Lee HW, Agrawal V, Kim YM, Kwon YG. Yes-associated protein regulates endothelial cell contact-mediated expression of angiopoietin-2. Nat Commun. 2015;6:6943.

\section{Publisher's Note}

Springer Nature remains neutral with regard to jurisdictional claims in published maps and institutional affiliations.
Ready to submit your research? Choose BMC and benefit from:

- fast, convenient online submission

- thorough peer review by experienced researchers in your field

- rapid publication on acceptance

- support for research data, including large and complex data types

- gold Open Access which fosters wider collaboration and increased citations

- maximum visibility for your research: over $100 \mathrm{M}$ website views per year

At BMC, research is always in progress.

Learn more biomedcentral.com/submissions 